\title{
Digital image correlation and infrared measurements to determine the influence of a uniaxial pre-strain on fatigue properties of a dual phase steel.
}

\author{
R. Munier ${ }^{1,2, a}$, C. Doudard ${ }^{1}$, S. Calloch ${ }^{1}$, and B. Weber ${ }^{2}$ \\ 1 Laboratoire Brestois de Mécanique et des Systèmes (L.B.M.S.), ENSIETA - 2 rue François Verny - \\ 29806 Brest Cedex 9, France \\ 2 ArcelorMittal Maizières Research and Development, BP 30320 - F-57283 Maizières-les-Metz Cedex, \\ France
}

\begin{abstract}
The high cycle fatigue (HCF) is a major element for a great design of automotive parts. A wide part of the steel sheets for the automotive industry are stamped, sometimes deeply. During this operation, the steel is plastically strained in different directions, so that a good prediction of the fatigue behavior requires the determination of the fatigue properties of the pre-strained material. Nowadays, the evolution of HCF properties is often neglected, because of prohibitive time dedicated to traditional fatigue campaigns. To reduce the characterization time, self-heating measurements are used. This approach permits to identify the influence of homogeneous pre-strain on fatigue properties. The aim of this paper is to develop an original experimental test to identify this influence for a wide range of pre-strain with only one specimen. The study of a particular case of specimen with a constant gradient of pre-strain is presented. Digital image correlation is a way to determine the heterogeneity of the plastic pre-strain on the specimen and infrared measurements with a "1D" approach allows the determination of the influence of a plastic pre-strain on the fatigue properties of the studied steel.
\end{abstract}

\section{Introduction}

Structures and chassis parts of a vehicle are designed for a Low Cycle or for a High Cycle Fatigue mission. Concerning the high cycle fatigue, automotive components are obtained from high strength steel sheets by primary stamping operations. During these drawing operations, the steel is severely strained according to different directions or strain paths inducing thickness variations and cumulated plastic strain variations. In other words, the material has changed and the fatigue properties of the prestrained steel are no more the same than the as received ones. Figure 1 and figure 2 show the change of the fatigue properties between the initial dual phase steel and the dual phase steel pre-strained at a level of $20 \%$, for a zero mean stress $\Sigma_{m}$. The evolution of the fatigue properties is often neglected in the industry, mainly because of the prohibitive time which is dedicated to traditional fatigue campaigns. For example, 25 specimens and one month at a load frequency $f_{r}$ of $30 \mathrm{~Hz}$ are necessary to plot a Wöhler curve that gives the number of cycle relating the stress amplitude. To considerably reduce the time of characterization, self-heating measurements are used.

For several years, different working groups are using self-heating measurements [1] [2] [3] [4] [5]. The principle consists in following the temperature evolution of the specimen during cyclic loadings. Successive series of sinusoidal loading, under the yield stress, are applied on a specimen. At each level of stress, the mean stabilized temperature $\bar{\theta}$ is pointed out after 6000 cycles (figure 3 ). The

a e-mail: remi.munier@ensieta.fr 


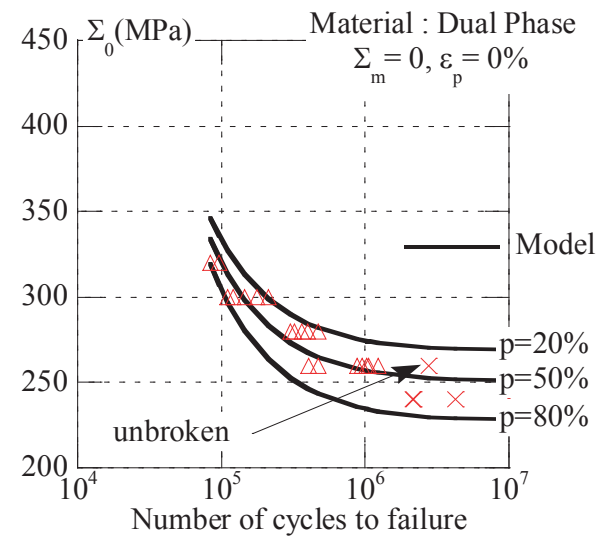

Fig. 1. Wöhler curve of the DP600 steel without pre-strain.

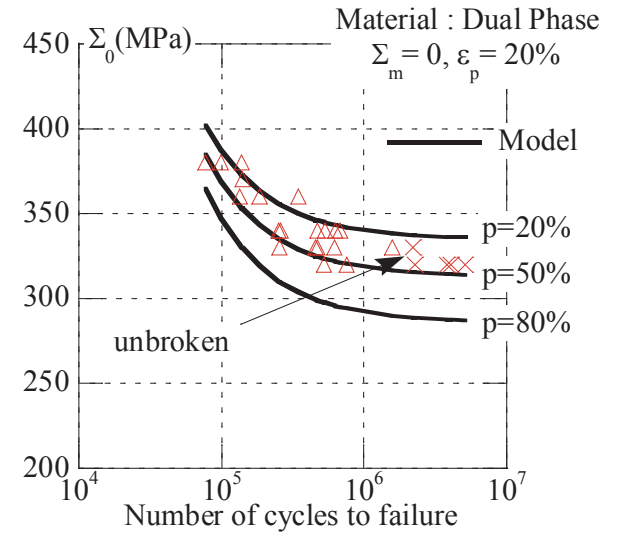

Fig. 2. Wöhler curve of the DP600 steel prestrained at a level of $20 \%$.

temperature change is due to microplasticity activation. Finally, a self-heating curve, relating the temperature change to the applied stress amplitude $\Sigma_{0}$, can be plotted. One observes that beyond a given stress level, that is closed to the fatigue limit, the steady-state temperature increase significantly (Figure 4). Recently, a two-scale probabilistic model has been proposed to identify HCF properties from self-heating measurement [6].

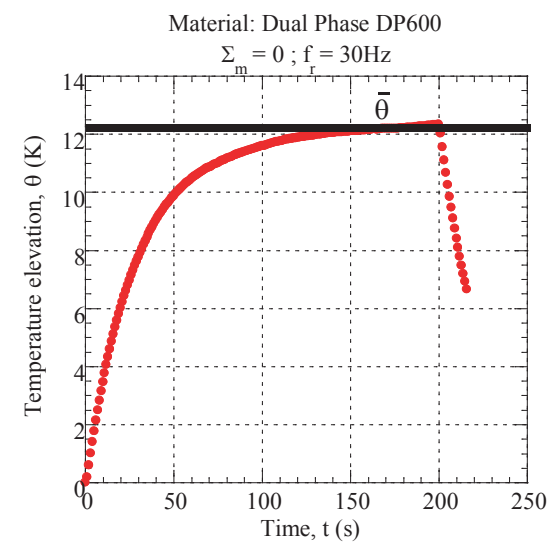

Fig. 3. Temperature elevation during a cyclic loading.

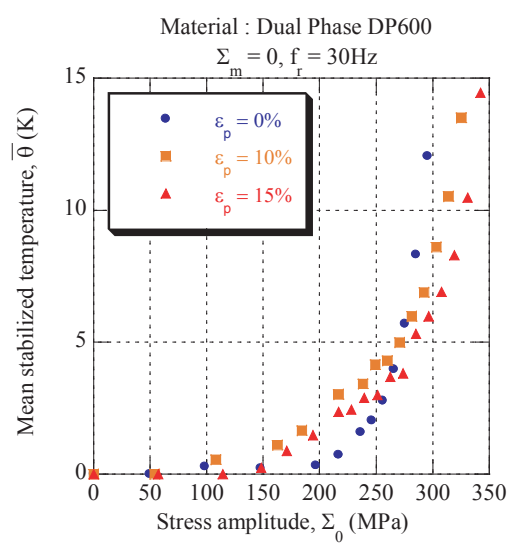

Fig. 4. Self-heating curves of a pre-strained Dual Phase DP600 steel.

The influence on the fatigue properties of the studied steel can be determined by self-heating measurements by using specimen with a constant section that have been uniformerly pre-strained (figure 4). Ones observes that the increase of temperature is delayed by the applied pre-strain (i.e., an improve of fatigue properties). According to the results obtained from classical fatigue campaigns and from self-heating measurements, a linear influence of the pre-strain on the endurance limit is considered [7]

$$
\bar{\Sigma}_{\infty}\left(\varepsilon^{p}\right)=\left(1+\beta \varepsilon^{p}\right) \bar{\Sigma}_{\infty}(0 \%),
$$

with $\beta$ the parameter that drives the linear influence of the plastic pre-strain. Specimens with a constant section allow the identification of the parameter $\beta$ that drives the influence of a plastic pre-strain on the fatigue properties. Nevertheless, this approach requires a specimen per level of plastic strain. It is proposed, in this paper, to develop a particular specimen to identify this influence for a wide range of pre-strain with one specimen. 
This paper is divided into two main sections. In the first one, the geometry of a specific specimen [8] which permits to obtain a constant gradient of pre-strain is determined. The pre-strain test is then performed. To identify the level of plastic strain that has been reached in the gauge section of the specimen, digital image correlation is used. The second section deals with a $1 \mathrm{D}$ approach for selfheating measurements. The principle of the resolution of the 1D heat equation conduction with the influence of a plastic pre-strain will be presented. To take into account the heterogeneities of the pre-strained specimen, an infrared camera is required. The comparison with the identification results of HCF properties change obtained from specimens with a constant section validates the proposed approach.

\section{Pre-straining test of a specific specimen}

The studied material is a dual phase steel sheet so that the thickness of the proposed specimen is constant $(2.55 \mathrm{~mm})$. The half-width $\mathrm{b}(\mathrm{x})$ of this specimen is chosen to obtain a constant pre-strain gradient.

\subsection{Geometry of the specific specimen}

To determine the half-width $\mathrm{b}(\mathrm{x})$ of the specimen, the plastic incompressibility of the steel is used. With $S_{0}(x)$ the initial section, $\mathrm{S}^{\prime}(\mathrm{x})$ the section after pre-straining and $\mathrm{u}(\mathrm{x})$ the displacement of this section

$$
\frac{S_{0}(x)}{S^{\prime}(x)}=1+\frac{u(x+d x)-u(x)}{d x}=1+\frac{d u(x)}{d x} .
$$

The true stress $\sigma_{V}(x)$ is then given by

$$
\sigma_{V}(x)=\frac{F_{0}}{S^{\prime}(x)}=\frac{F_{0}}{S_{0}(x)} \times \frac{S_{0}(x)}{S^{\prime}(x)}=\frac{F_{0}}{2 b(x) e_{0}} \times\left(1+\frac{d u(x)}{d x}\right),
$$

with $F_{0}$ the maximum strength applied during the pre-strain test and $e_{0}$ the initial thickness of the sheet. To find the expression of the displacement, the hypothesis of a constant gradient $\mathrm{K}$ of plastic strain obtained after pre-straining are considered

$$
\epsilon_{p}(x)=K(x+u(x)),
$$

with

$$
\epsilon_{p}(x)=\ln \left(1+\frac{d u(x)}{d x}\right)
$$

It leads to a differential equation that can be solved. The displacement $\mathrm{u}(\mathrm{x})$ is then given by

$$
u(x)=-x-\frac{1}{K} \ln (1-K x) .
$$

To complete the analytical formulation of the half width $b(x)$, an approximation on the plastic behavior law of the steel is determined (figure 5) and finally

$$
b(x)=\frac{F_{0}}{2 e_{0} \sigma_{V}(x)} \frac{1}{1-K x} .
$$

The value of the parameter $\mathrm{K}$ is defined by the maximum of plastic pre-strain that will be reach at the center of the specimen. In order to have a valid approximation of the behavior law, the maximum value of pre-strain is $20 \%$. The profile of a symmetric specimen can be then calculated (figure 6).

Finite elements calculation can be made by using the profile of the specimen and the behavior law of the steel, in order to check the distribution of the plastic pre-strain on the specimen, with a displacement imposed (figure 7). We can observe a distribution by bands. Furthermore, a light localization of the strain can be observed at the center of the specimen. To avoid this phenomenon and keep a uniform value of strain along the width, only about $17 \%$ of plastic pre-strain will be reached during the pre-strain test. 


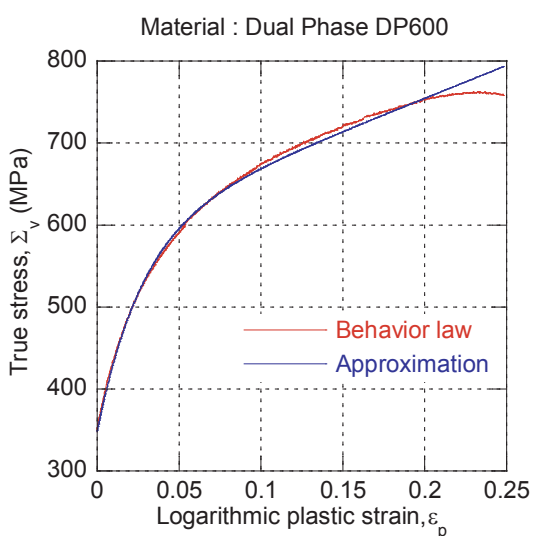

Fig. 5. Experimental results and identified behavior law of the DP600.

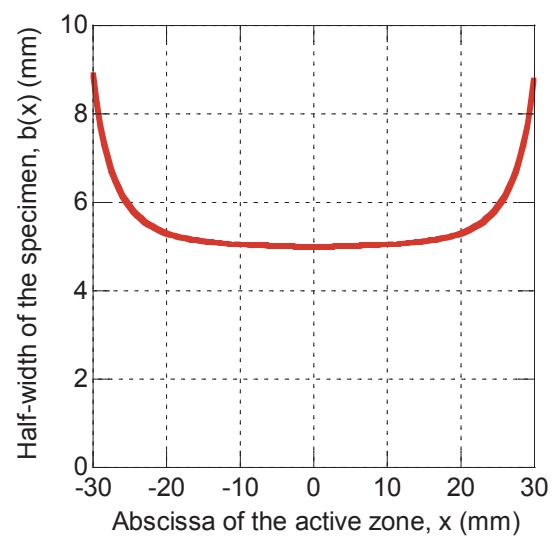

Fig. 6. Calculated profile of the specimen.
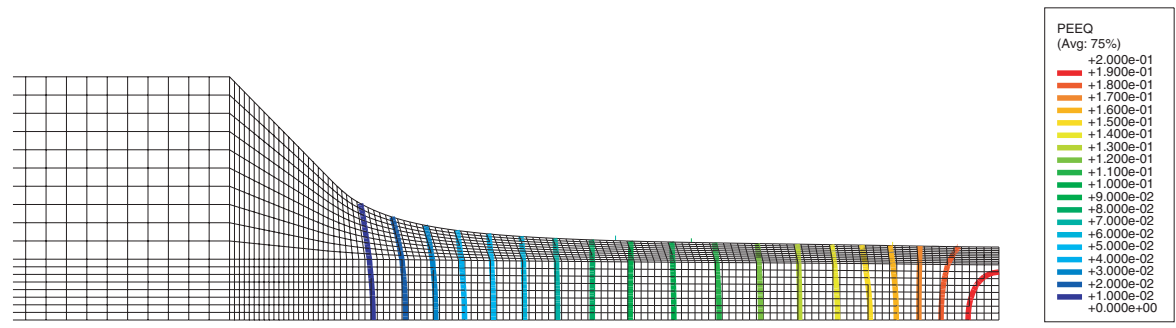

Fig. 7. Distribution of the plastic pre-strain on the specimen to reach $20 \%$ (PEEQ = cumulated plastic strain).

\subsection{Identification of the constant gradient of pre-strain by digital image correlation}

Digital correlation image is used, on the one hand, to validate the constant pre-strain gradient, and the other hand, to identify the experimental value of plastic pre-strain gradient. The difference between the theoretical and experimental value is due to the influence of tension machine rigidity on the mechanical response during the test.

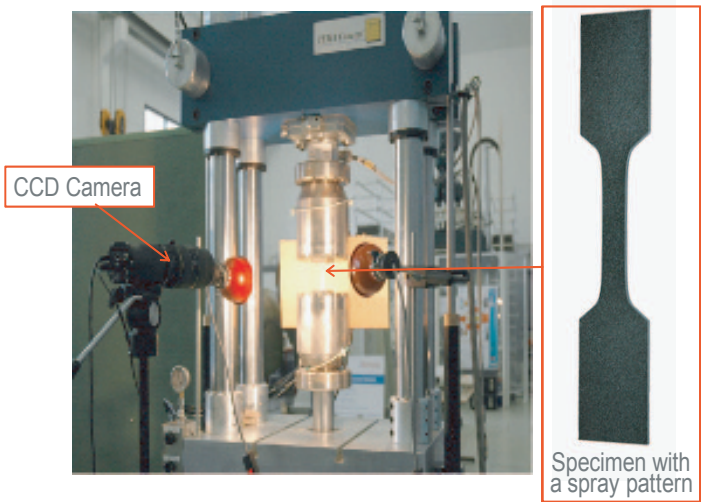

Fig. 8. Experimental device for the pre-strain test.

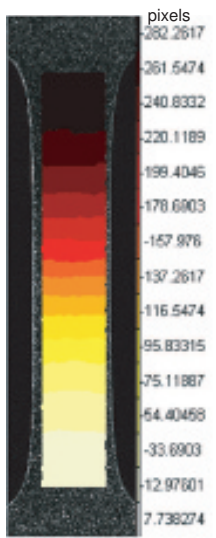

Fig. 9. Displacement (pixels) obtained with CorreliLMT.

The principle of digital image correlation consists in following the displacement of a specific zone on successive images taken by a CCD camera ( 8 million pixels). To ensure a good quality of the re- 
sults, a particular attention is paid to the contrast between the different pixels of the images. Thus, the specimen has a spray pattern (black paint with random white points). Software for displacement field measurements by digital image correlation, CorreliLMT [9], is used. The displacement field obtained at the end of the test is given in pixels (figure 9). The displacement is constant by bands. Indeed, by using the analytical expression of the displacement $\mathrm{u}(\mathrm{x})$, the gradient of plastic pre-strain $K_{i d}$ can be identified (figure 10).
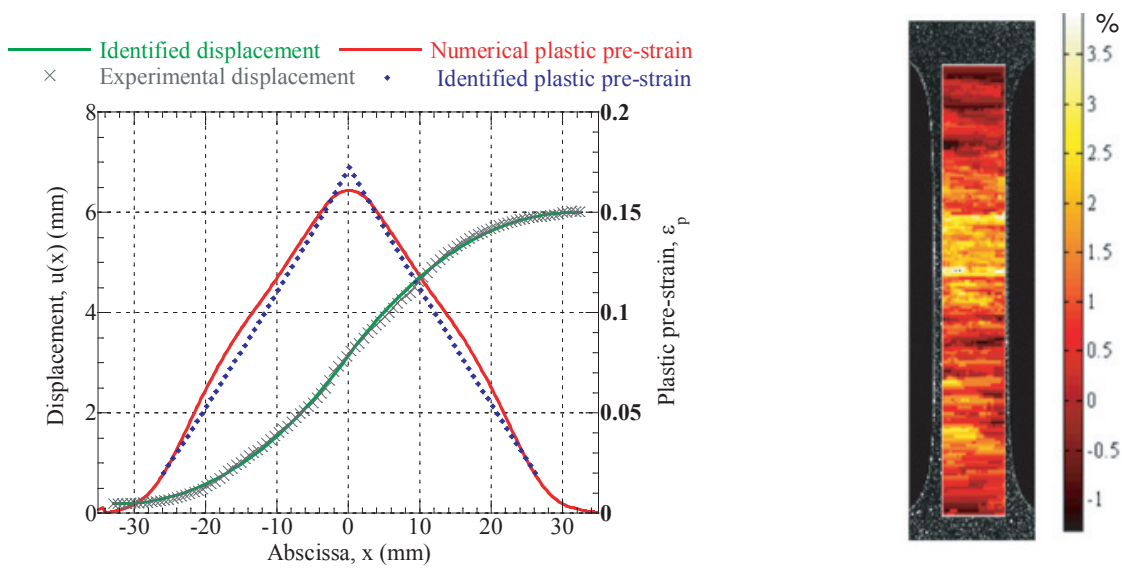

Fig. 10. Identification of the parameter $K_{i d}$ on the displacement and plastic strain obtained.

Fig. 11. Error map representing the difference (in $\%$ ) between the identified displacement and the experimental one.

To check the validity of the approximation, an error map can be plotted, representing the difference (in \%) between the analytical identification and the results obtained with CorreliLMT (figure 11). Finally, the identified parameter $K_{i d}$ gives information about the level of plastic pre-strain that has been reached during the pre-straining test. With the value of the experimental displacement really performed during the test, another FE computation can be performed using it as input data. The identified analytical distribution are in accordance with the numerical distribution of the plastic pre-strain (figure 10). The small differences obtained can be explained on the one hand by the boundary conditions that are not taken into account among the hypothesis and on the other hand by the approximation of the behavior law of the dual phase steel.

\section{Self heating measurements with a 1D approach}

To take into account the heterogeneities of the specimen (section variations inducing stress variations, plastic pre-strain distribution), a 1D approach with an infrared camera is required (Flir Systems MWIR 9705, resolution: $320 \times 256$ pixels, medium wave with a $3-5 \mu m$ spectral range) to analyze the selfheating measurement.

\subsection{Solving of the 1D heat conduction equation}

For a 1D thermal diffusion problem, the local heat equation conduction is written as [10]

$$
\rho C \dot{\theta}(x, t)-\lambda^{\prime} \frac{\partial^{2} \theta(x, t)}{\partial x^{2}}-\lambda^{\prime} \frac{\partial \theta(x, t)}{\partial x} \frac{\partial S(x)}{\partial x} \frac{1}{S(x)}=S_{t}(x, t),
$$

with $\rho$ the mass density, $\mathrm{C}$ the specific heat capacity, $\lambda^{\prime}$ the isotropic thermal conductivity and $S_{t}$ the heat source. Due to the noise of the thermal signal and the regularizing effect of heat diffusion, the reconstruction of heat source maps is still a difficult task. In this paper, to handle the inverse problem, 
the theoretical result of the heat source is used to build a theoretical temperature field from a particular basis proposed by [10]. In this case, the identification of the heat source consists in minimizing the difference between the calculated temperature and the experimental one. The considered basis for the determination of the theoretical temperature field is based on a combination of two bases: a Fourier basis completed with a two order polynomial. The two order polynomial describes the local behavior of the temperature and the components of the Fourier basis describe the local fluctuations of the signal. The stabilized temperature field is then expanded as follows

$$
\theta(x)=a x^{2}+b x+c+\sum_{k>0} \theta_{a k}^{\prime} \cos \left(\frac{2 k \pi x}{L}\right)+\theta_{b k}^{\prime} \sin \left(\frac{2 k \pi x}{L}\right),
$$

with a, b, c parameters that have to be identified and $\theta_{a k}^{\prime}$ and $\theta_{b k}^{\prime}$ functions of the components of the Fourier basis [10]. To include the influence of a plastic pre-strain, the modification of the intensity of the process, that drives the scenario of microplasticity appearance, leads to consider a thermal source

$$
S_{t}(x)=\delta f_{r} \frac{\Sigma_{0}(x)^{m+2}}{\left(1+\beta \epsilon_{p}(x)\right)^{m}},
$$

with $\delta$ a parameter and $\mathrm{m}$ the Weibull modulus [6].

The parameter $\mathrm{b}$ drives the dissymmetry of the temperature fields. As the symmetry can be made by considering the difference of the temperature, $b$ is often equal to 0 . With these information, the heat equation conduction can be projected on this basis and written as a matrix

$$
\left[\begin{array}{c}
S_{t 0} \\
S_{t a k} \\
S_{t b k}
\end{array}\right]=\lambda^{\prime}\left[\begin{array}{ccc}
-2\left(1+g_{0}\right) & \omega_{k} \frac{h_{b k}}{2 L} & -\omega_{k} \frac{h_{a k}}{2 L} \\
-2 g_{a k} & \omega_{k}^{2}+\sum \frac{\omega_{j}}{2 L}\left(h_{b j-k}+h_{b j+k}-h_{b k-j}\right) & -\sum \frac{\omega_{j}}{2 L}\left(h_{a j-k}+h_{a j+k}-h_{a k-j}\right) \\
-2 g_{b k} & \sum \frac{\omega_{j}}{2 L}\left(h_{a j-k}-h_{a j+k}+h_{a k-j}\right) & \omega_{k}^{2}-\sum \frac{\omega_{j}}{2 L}\left(-h_{b j-k}+h_{b j+k}-h_{b k-j}\right)
\end{array}\right]\left[\begin{array}{c}
a \\
\theta_{a k}^{\prime} \\
\theta_{b k}^{\prime}
\end{array}\right]
$$

with $\mathrm{L}$ the length of the active zone of the specimen, $\mathrm{k}$ the number of components of the Fourier basis, $\omega_{k}=2 k \pi / L$ and $g_{i}$ and $h_{i}$ are the components on the Fourier basis of the functions

$$
h(x)=\frac{\partial S(x)}{\partial x} \frac{L}{S(x)} \text { and } g(x)=\frac{\partial S(x)}{\partial x} \frac{x}{S(x)} .
$$

Finally, by entering initial values of the parameters in the thermal sources ( $\mathrm{m}$ and $\beta$ ), and with an inversion of the previous matrix, a temperature can be calculated. By successive iterations, the adjustment of the parameters will be made to minimize the difference between the calculated temperature and the experimental one and the value of $\beta$ will give the influence of the plastic pre-strain.

\subsection{Results}

Some precautions have to be taken with the use of one infrared camera. The surface of the specimen has to be painted with a dark paint in order to obtain a high constant emissivity. Then, an infrared measurement is made and the surrondings of the specimen have to be protected in order to avoid the stray radiations. The experimental dispositive is presented on the figure 12. Self-heating measurement can be made with successive series of loading. One film corresponds to one level of stress. On each film, the detection of the stabilized temperature is made (figure 13).

For each film, a meaning along the width is made, the difference between the experimental temperature and the calculated one is minimized and the values of the parameters are identified. For example figure 14 shows the results of the minimization and the differences between the experimental and identified temperature fields are very weak. As previously, it is possible to consider an error map on the studied zone to ensure the uniformity of the temperature (figure 15). The error is less than $3 \%$ which 


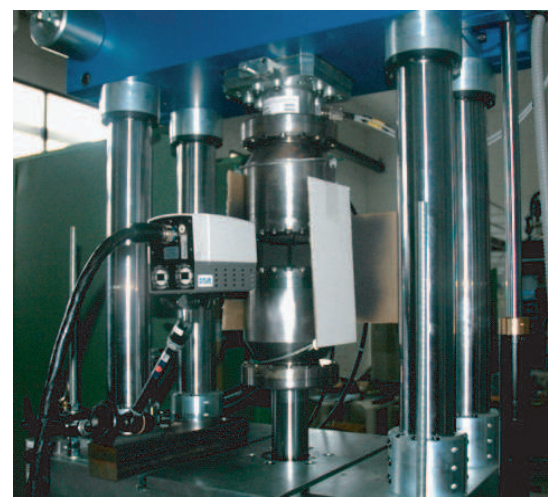

Fig. 12. Experimental device for infrared measurments.

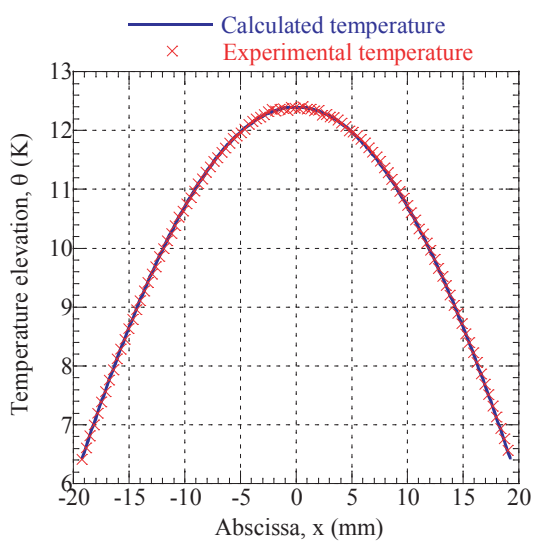

Fig. 14. Minimization between the calculated temperature and the experimental one.

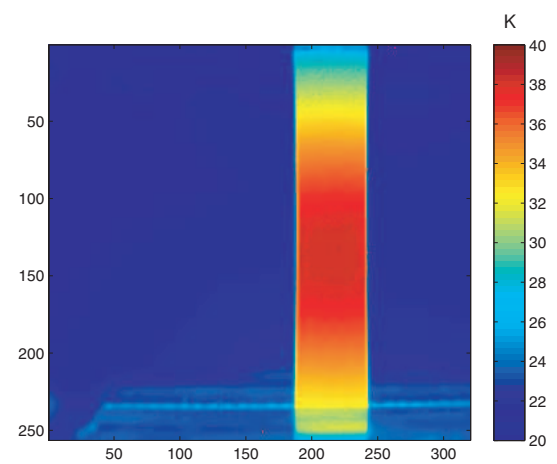

Fig. 13. Image issue from the IRcamera.

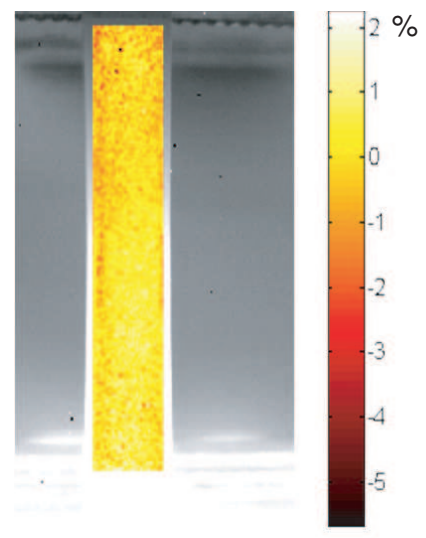

Fig. 15. Error map representing the differences (in $\%$ ) between the experimental temperature and the identified one.

validates the expression of the calculated temperature.

Finally, the influence of a plastic pre-strain is identified and compared with the values obtained on specimens with a constant section that have been pre-strained in tension mode [7] (table 1). A good agreement is obtained.

Table 1. Comparison between the values of the parameter $\beta$ obtained.

\begin{tabular}{ll}
\hline Specimen with a specific profile & Pre-straining in traction mode \\
\hline$\beta=1.34$ & $\beta=1.3$ \\
\hline
\end{tabular}

\section{Conclusions and perspectives}

In this study, two experimental techniques are put in place. Digital image correlation allows to validate the design of the specific specimen and to identify the value of the constant plastic strain gradient. 
Self-heating measurements with an infrared camera allows the determination of the heat source field of heterogeneous pre-strained specimen.

The challenge of this study was to determine the influence of a uniaxial plastic pre-strain on HCF properties for the studied dual-phase steel. By using a specific specimen and a 1D approach to handle the reconstruction of heat source maps, the identification of plastic pre-strain influence is performed on a wide range (from $6 \%$ to $17 \%$ ). The value of the $\beta$ parameter obtained with this approach is very close to the one determined with traditional fatigue campaigns which validates the approach.

Finally, with only one specimen and only on self-heating measurement, this method allows the determination of the influence of a uniaxial plastic pre-strain on the fatigue properties of a DP600 steel. Every experimental technique and every tool for the treatment of the datas have been validated so that it is possible to apply this approach for others steel grades.

\section{References}

1. M. P. Luong "Infrared Thermography of Fatigue in Metals," SPIE 1682 ,(1992) 222-233.

2. G. La Rosa and A. Risitano "Thermographic Methodology for Rapid Determination of the Fatigue Limit of Materials and Mechanical Components", Int. J. Fat. 22 [1],(2000) 65-73.

3. P. K. Liaw, H. Wang, L. Jiang, B. Yang, J. Y. Huang, R. C. Kuo and J. C. Huang (2000) "Thermographic detection of fatigue damage of pressure vessel at $1 \mathrm{~Hz}$ and $20 \mathrm{~Hz}$ ", Scripta Materialia 42 [4], 389-395.

4. A. Galtier, O. Bouaziz and A. Lambert "Influence de la Microstructure Des Aciers sur leurs Propriétés Mécaniques," Méc. Ind. 3 [5], (2002) 457-462.

5. C. Doudard, S. Calloch, F. Hild, P. Cugy and A. Galtier "Identification of the scatter in high cycle fatigue from temperature measurements," C.R. Mécanique 332 [10], (2004) 795-801.

6. C. Doudard, S. Calloch, P. Cugy, A. Galtier and F. Hild "A probabilistic two-scale model for highcycle fatigue life predictions," Fat. Fract. Eng. Mat. Struct. 28,(2005) 279-288.

7. C. Doudard, "Détermination rapide des propriétés en fatigue à grand nombre de cycles à partir d'essais d'auto-échauffement", PhD ENS Cachan, (2004).

8. Kaszynski P., "Indentation Cyclique et Déformation Progressive”, PhD E.N.S. Cachan, (1999).

9. Chevalier L., Calloch S., Hild F., Marco Y., ’Digital image correlation used to analyse the multiaxial behavior of rubberlike materials", Eur J Mech A Solid, 20, 2, (2001) 168-187.

10. Doudard C., Calloch S., Hild F. and Roux S. "Identification of heat source fields from infra-red thermography: Determination of 'self-heating' in a dual-phase steel by using a dog bone sample", Mechanics of Materials 42 1, (2010) 55-62. 